\title{
Beneficial effects of ambiguous precues: Parallel motor preparation or reduced premotoric processing time?
}

\author{
INES JENTZSCH,${ }^{\mathrm{a}}$ HARTMUT LEUTHOLD,${ }^{\mathrm{a}}$ AND K. RICHARD RIDDERINKHOF ${ }^{\mathrm{b}, \mathrm{c}}$ \\ ${ }^{a}$ Department of Psychology, University of Glasgow, Scotland, UK \\ ${ }^{\mathrm{b}}$ Department of Psychology, University of Amsterdam, Amsterdam, The Netherlands \\ ${ }^{\mathrm{c}}$ Department of Psychology, Leiden University, Leiden, The Netherlands
}

\begin{abstract}
The present study was designed to investigate the mechanisms underlying movement preprogramming in situations where informative but ambiguous precue information is used. In a response precuing task that involved flexion or extension movements with the right or left index finger, a spatially compatible precue conveyed partial information about side, about direction, no parameter information (ambiguous condition), or no information at all. Advance movement preparation was indicated by reaction-time shortening for all informative precue conditions. The analysis of stimulus- and response-locked lateralized readiness potential onsets revealed a clear and exclusive motoric origin of the ambiguous-precue benefit. Additional analyses ruled out a strategic trial by trial choice of just one of the two ambiguous alternatives and provided evidence for a parallel preparation of both response alternatives when information only about direction or ambiguous precue information is provided.
\end{abstract}

Descriptors: Movement preparation, Precuing paradigm, Lateralized readiness potential, LRP, Contingent negative variation, $\mathrm{CNV}$, Ambiguous precues

In the response precuing paradigm (Rosenbaum, 1980, 1983), subjects are to prepare for a discriminatory motor response on the basis of specific advance information about the nature of the required action. When the precued information fully or partially specifies the parameters of the upcoming motor act, the response is issued more rapidly compared to when the precue provides no specific advance information about response parameters. Curiously, such a response-time benefit is also observed when precues provide informative but ambiguous advance information, that is, when the number of possible response alternatives is reduced without specifying any movement parameter. In the present study, we analyzed event-related brain potentials (ERPs) associated with movement preparation in motor cortex to determine whether the beneficial effect of ambiguous precues involves (1) motoric or premotoric stages of response preparation, and (2) advance preparation for a specific response (based on, e.g., guessing) or simultaneous preparation for multiple responses.

We thank two anonymous reviewers for their helpful comments on a previous version of the manuscript. This research was supported by a Postdoctoral Research Fellowship to Ines Jentzsch from ESRC (Ref: T026 27 1375) and a Grant to Hartmut Leuthold from the Royal Society (Ref: 23524).

Address reprint requests to: Ines Jentzsch, University of Glasgow, Department of Psychology, 58 Hillhead Street, Glasgow, G12 8QB, Scotland, UK. E-mail: i.jentzsch@psy.gla.ac.uk.
It is commonly known that optimal motor performance is achieved by preparing in advance the upcoming movements as much as possible. Thus, a sprinter will increase his/her chances to win a race if he/she starts with the shortest possible delay. To this end, athletes not only aim at anticipating exactly the time of the starting shot but also to completely prepare the actual motor act. Preparation of simple motor acts has been examined by students of motor control, who assume that a limited number of movement parameters is specified in a so-called motor program before the motor act is actually executed (e.g., Keele, 1981). This prespecification of the motor program is believed to be responsible for savings in reaction time (RT) as compared to when no such preparation occurred before the execution of the movement. Not all researchers share, however, the idea of a motoric locus of this RT benefit, arguing rather that the preselection of responses at a nonmotor level is effective (e.g., Goodman \& Kelso, 1980). Yet it is largely unclear to what extent motor processes contribute to RT savings even under such task conditions.

The parameter-specific preparation of movements has been studied with the response precuing paradigm (Rosenbaum, 1980, 1983). Here, responses vary on more than one dimension (e.g., hand, direction, extent) and a precue conveys advance information about all, some, or no movement parameters. For example, in a trial where participants had to perform a forward movement to a near target with the left hand, a symbolic precue (letters) informed them in advance about the response side (e.g., 
$\mathrm{LXX}=$ left). The response signal then delivered the remaining information (e.g., LFN = left-forward-near). RT was found to decrease with the amount of precuing information. More important, RT was reduced most for hand, less for direction, and least for extent information. On the basis of these differential RT benefits, Rosenbaum (1980) attributed the precuing effect to savings in motoric processing time resulting from the movement preprogramming that takes place during the foreperiod.

Goodman and Kelso (1980) criticized the precuing method, however, arguing that the observed RT savings do not reflect a genuine motor effect, but rather the facilitation of stimulusresponse (S-R) translation processes at a premotoric level (response selection). Thus, time-consuming S-R translation processes might have been involved in Rosenbaum's experiments because the mapping between stimuli and associated responses was symbolic rather than spatial. In a first step, Goodman and Kelso tested the translation hypothesis by contrasting results obtained for the symbolic S-R mapping with those for a spatially compatible S-R mapping. As expected, parameter-specific precuing effects (e.g., longer RT for specification of hand than direction) were present only with the symbolic mapping but were eliminated with the spatially compatible mapping. The translation hypothesis received further support from results obtained with the so-called ambiguous precue condition. Here, the precue reduced the number of S-R alternatives from eight to two but did not specify any specific parameter, thereby precluding motor program specification (Goodman \& Kelso, 1980). For example, the ambiguous precue announced that either a left-forward-far or a right-backward-near movement was forthcoming, with the two $\mathrm{S}-\mathrm{R}$ alternatives differing in all three parameters. Nevertheless, the ambiguous precue produced a precuing effect indistinguishable from the one obtained in conditions in which two specific movement parameters were unequivocally prespecified (e.g., leftforward but extent unknown). Goodman and Kelso therefore concluded that any precue information merely reduces the number of S-R alternatives, thereby shortening the response selection stage before motoric processing.

Yet, the validity of this conclusion depends critically on the assumption that ambiguous precues shorten only response selection but not motoric processing time. This assumption is shared by many studies of motor programming using ambiguous precue conditions to control for the confound of number of precued movement dimensions and the number of S-R alternatives (e.g., Lépine, Glencross, \& Requin, 1989; Vidal, Macar, \& Bonnet, 1996; Wright, Black, Park, \& Shea, 2001). Larish and Frekany (1985; see also Larish, 1986; Rosenbaum \& Kornblum, 1982) were among the few researchers considering the possibility that RT savings with (ambiguous) precues may result from multiple preparation of responses at the motoric level. According to this hypothesis of multiple motor preparation, ambiguous responses might be completely programmed in advance of the imperative response signal, especially when only a small number of S-R alternatives (e.g., two-choice RT) remain as in the experiment of Goodman and Kelso (1980). Despite the possibility that parallel motor preparation is responsible for the shortening of motoric processing time, to our knowledge, the locus of RT benefits induced by ambiguous precues has not undergone a rigorous test.

It is therefore of major theoretical interest to address the question whether RT savings with ambiguous precues reflect a genuine premotoric effect or a motoric effect due to multiple preparation of responses. Unfortunately, it is difficult to settle this issue with the traditional behavioral RT measure, basically because the motoric portion of RT is not directly observable. The present study therefore addresses this issue by taking advantage of the lateralized readiness potential (LRP), which has been used to resolve the controversy about the nature of the standard precuing effect (Leuthold, Sommer, \& Ulrich, 1996; Osman, Moore, \& Ulrich, 1995).

The LRP provides a specific index to trace the time course of motor activation (cf. Coles, Gratton, \& Donchin, 1988; De Jong, Wierda, Mulder, \& Mulder, 1988; Miller \& Hackley, 1992) as generated within the primary motor cortex (MI; Leuthold \& Jentzsch, 2002a; Osman \& Moore, 1993). The locus of experimental effects can be inferred by analyzing LRP onsets obtained in waveforms time-locked either to response signal onset or to the overt response (Leuthold et al., 1996; Osman \& Moore, 1993). The interval from response signal onset to stimulus-locked LRP onset (S-LRP interval) indicates the duration of those processes occurring before the start of the LRP. The interval between response-locked LRP onset and the overt response (LRP-R interval) indicates the duration of those processes that occur after LRP onset. That is, the S-LRP and the LRP-R intervals can be used as chronometrical markers for premotoric and motoric processing, respectively.

Initial evidence that an ambiguous precue might shorten premotoric as well as motoric processing time has been provided by Osman et al. (1995). The authors used a precuing paradigm where precues that preceded the response signal provided partial information about the parameter finger (index or middle finger), hand (left or right), or ambiguous information (e.g., left index and right middle finger), all these precue conditions reducing the number of S-R alternatives from four to two. The noninformative precue delivered no advance information. There was a reliable precuing effect due to faster responses with partial and ambiguous precues than with noninformative precues. Because partial and ambiguous precues did not differ in RT from each other, Osman et al.'s RT findings seem to suggest a response selection locus of the precuing effect (e.g., Goodman \& Kelso, 1980; Larish \& Frekany, 1985). Interestingly, however, the analysis of S-LRP and LRP-R intervals revealed a shortening not only of premotoric processing time but also of motoric processing time when both partial and ambiguous precues were presented as compared to the noninformative condition. In our opinion, Osman et al.'s (1995) results provide initial evidence for the view that like partial precues, ambiguous precues shorten motoric processing time. It remains unclear, however, whether or not the motoric RT benefit with the ambiguous precue is due to multiple motor preparation of the indicated response alternatives.

The present study attempts to provide a refined test of the locus of RT savings with an ambiguous precue condition and of its underlying mechanism. In the present study, we replaced the parameter finger as used by Osman et al. (1995) by the parameter movement direction for two reasons. First, this has the advantage of better comparability to previous LRP studies of preprogramming that used these motor parameters and revealed, at least to some extent, a motoric locus of the precuing benefit with movement direction (Leuthold et al., 1996; MüllerGethmann, Rinkenauer, Stahl, \& Ulrich, 2000). Second, it extends the study of Osman et al. in a theoretically important way. Thus, it is possible that the preparation of effectors reflects the organization of the underlying anatomy (cf. Wild-Wall, 
Sangals, Sommer, \& Leuthold, 2003) allowing more easily for multiple motor preparation (see also Rosenbaum \& Kornblum, 1982). By contrast, such preparation might be precluded for the parameter direction, because here antagonistic movement values need to be coded. It is therefore of interest to know whether the LRP findings of Osman et al. generalize to this movement parameter.

\section{Objective 1: Locus of Precuing Effects}

Our first major aim, then, was to examine the locus of the precuing effect in the present experiment using an ambiguous precue condition. As in earlier LRP precuing studies (e.g., Leuthold et al., 1996), processing benefits in premotoric processing stages would be revealed by a shortening of the SLRP interval. In the present study, we use a highly compatible S$\mathrm{R}$ mapping to reduce premotoric precuing benefits in $\mathrm{RT}$. Nevertheless, if the view of a merely premotoric locus of precuing effects holds true (e.g., Goodman \& Kelso, 1980), we would expect to find S-R processing benefits only in this premotoric portion of RT. Importantly, however, if precues also induce a shortening of motoric processing time, this should be revealed by a significantly shorter LRP-R interval as compared to the noninformative precue condition (Leuthold et al., 1996; MüllerGethmann et al., 2000; Osman et al., 1995).

To reveal possible influences on motor programming during the foreperiod, we will also investigate the behavior of the contingent negative variation (CNV), a movement-related potential normally associated with abstract parameter selection. Ulrich, Leuthold, and Sommer (1998) showed the late CNV, a centroparietally dominant negativity during the foreperiod reflecting motor preparation (cf. Rohrbaugh \& Gaillard, 1983), to gradually increase with the number of precued movement dimensions (see also MacKay \& Bonnet, 1990). That is, foreperiod CNV amplitude was largest when the precue conveyed full information, it was intermediate when hand and direction or hand and force were precued, and smallest when only hand information was available. Ulrich et al. (1998) concluded that the foreperiod $\mathrm{CNV}$ might reflect abstract programming at a central level, where all movement parameters might be prepared independently. Because the ambiguous precue is presumed to reduce the number of $\mathrm{S}-\mathrm{R}$ alternatives, this condition should elicit a foreperiod CNV similar to the one obtained with the noninformative precue, whereas foreperiod $\mathrm{CNV}$ amplitude for partial hand and direction precues should be enlarged.

\section{Objective 2: Multiple motor preparation and strategic influences}

Our second major objective was to examine the hypothesis of multiple motor preparation (cf. Larish \& Frekany, 1985). It is important to note that in the ambiguous and direction precue conditions, in principle, participants can prespecify the movements in both hands during the foreperiod. Assuming that the left and right hands are controlled by separate motor programs (e.g., Cardoso de Oliveira, 2002), it is conceivable that two motor programs, one for each hand, are compiled and both hands are simultaneously activated. Recently, we have found that parallel activation of two motor program elicits a larger foreperiod $\mathrm{CNV}$ than activation of a single motor program (Jentzsch \& Leuthold, 2002). Accordingly, the finding of a larger foreperiod CNV for the ambiguous precue than for a partial precue specifying response hand would support the multiple motor preparation hypothesis.
More importantly, analysis of foreperiod CNV activity from lateral recording sites will allow us to more directly reveal the simultaneous activation of both hands for the ambiguous and direction precues. Using a standard precuing task, we have recently shown parallel preparation of the two hands when only movement direction was precued but response hand was still unknown (Leuthold \& Jentzsch, 2001). More specifically, direction precues elicited CNV activity of identical magnitude over lateral MI recording sites. We then compared the magnitude of this ipsilateral and contralateral CNV activity with activity elicited in the full precue condition that delivered additional information about hand. As expected, CNV activity in the full precue condition was larger contralaterally than ipsilaterally to the prepared hand. The comparison with the direction precue revealed that $\mathrm{CNV}$ activity over contralateral recording sites was of the same size in both conditions. More importantly, a reliably larger activity over ipsilateral recording sites was found in the direction precue than in the full precue condition. From these results we inferred that with partial information about movement direction, participants prepared both left and right hand responses in parallel as indicated by the same level of motor activity as in the full precue condition. Here we will apply the same comparisons of CNV activity over lateral motor cortices to test the notion of multiple preparation in direction and ambiguous precue conditions.

Alternatively, instead of preprogramming mutually exclusive parameter values, subjects might adopt a guessing strategy by preprogramming in full on each trial only one of the two possible movements in the ambiguous precue condition, also leading to an overall RT benefit. To rule out such a major strategic contribution, we will analyze in more detail data resulting for different RT categories. More specifically, all trials will be split into three portions of fast, medium, and slow trials depending on RT in each trial separately for each precue condition and participant. Suppose for a moment that participants, when provided with an ambiguous precue, did not prepare both response alternatives (e.g., left extension and right flexion) but only one response alternative in full (e.g., right flexion). In this case, one should expect that when participants correctly anticipated the forthcoming response (right flexion), this should result in fast responses, reflecting the benefit of having programmed a movement with full advance information. By contrast, when participants guess and prepare the incorrect response (left extension), this should result in much longer RT due to the necessary reprogramming of response parameters (e.g., Larish \& Frekany, 1985; Leuthold \& Jentzsch, 2002b). Crucially, due to full advance preparation, a negative foreperiod LRP should show up with fast trials whereas slow trials should show a positive LRP activation, indicating preparation of the incorrect response hand. Note that this prediction holds true for both the partial direction and the ambiguous precue condition.

The present study used a response precuing task involving flexion or extension movements with the left or right index finger. Participants were provided either with advance information about hand or direction or with ambiguous information. In addition a noninformative precue, leaving all four response alternatives unspecified was used. As recommended in previous precuing studies (Goodman \& Kelso, 1980; Larish \& Frekany, 1985), the mapping of precues and response signals to responses was spatially compatible. In contrast to Osman et al.'s (1995) design, we used a horizontal instead of a vertical arrangement in order to maximize S-R compatibility (e.g. Nicoletti, Umiltà, 
Tressoldi, \& Marzi, 1988), thereby minimizing the amount of time needed for S-R translation processes at the response selection stage (Sanders, 1980, Teichner \& Krebs, 1974).

Lateral presentation of stimuli can be problematic when measuring LRP onsets, because lateralized stimuli are known to produce more negativity in the visual cortex contralateral to the stimulus side (e.g., Hillyard \& Münte, 1984). This asymmetric negativity occurs at about $200 \mathrm{~ms}$ after stimulus onset, making it possible for this sensory activity to overlap with the LRP and mimic a phasic activation of the correct hand (cf. Eimer, 1998). This sensory effect is not especially problematic for the LRP observed during the foreperiod, because this activation decays quite rapidly. However, as mentioned by Leuthold et al. (1996), a more serious problem concerns the timing of stimulusand response-locked LRP onsets. Because the early asymmetric sensory ERP activity emerges at a constant time after stimulus presentation, S-LRP onsets might reflect the onset of asymmetric sensory activity rather than motoric activity. Moreover, sensory activity might also distort LRP-R onset effects, because stimulus-locked lateralized ERP activity is not necessarily eliminated when response-locked LRPs are computed. However, sensory influences should be attenuated due to the response-locked averaging of waveforms; hence, the overlap problem appears to be especially virulent when a low onset criterion is applied.

In the present experiment, we tried to control for the impact of sensory activity on LRP onset in several ways. First, lateral stimuli were presented as close to fixation as possible in an attempt to reduce asymmetric sensory activity. Second, we measured LRP onsets by using different onset criteria to check the stability of our results. Third, we recorded the ERP from 70 electrode sites, giving us the chance to model the source of this parietal sensory activity and using the primary motor cortex source waveforms to measure LRP onset latencies. Basically, one can describe such a lateralized motor activity measure in terms of a dipole-corrected LRP, for which constant and responseunrelated sensory influences are minimized.

In summary, this study examines the locus and mechanism underlying RT savings in the response precuing paradigm. Specifically, we test the assumption that ambiguous precues shorten the premotoric portion of RT by reducing the number of S-R alternatives (e.g., Goodman \& Kelso, 1980). This assumption would receive support from the finding of an earlier S-LRP onset in the ambiguous than in the noninformative precue condition. In addition, because motor parameters are assumed not to be prespecified in the ambiguous and noninformative precue conditions, the foreperiod $\mathrm{CNV}$ is expected to be of identical amplitude in those conditions, while being smaller as compared to the other partial precue conditions. By contrast, the multiple motor preparation account predicts a foreperiod $\mathrm{CNV}$ that is larger for ambiguous than for noninformative precues and might even exceed the one observed for the hand precue. More importantly, if ambiguous and direction precues lead to simultaneous activation of the two response hands, ERP activity recorded over left and right motor cortices should be as large as contralateral and larger than ipsilateral ERP activity elicited by the hand precue. Finally, a strategic effect on motor preparation, in a way that participants guess and fully prepare just one of the two remaining movement alternatives with ambiguous and direction precues, is indicated in RT-classified LRP waveforms by a negative foreperiod LRP for fast trials and a positive LRP for slow trials.

\section{Method}

\section{Participants}

Eight women and four men (mean age: 31.0 years; range: 22 to 41 years, $S D=6.2$ ) participated in a single session lasting about $2 \mathrm{~h}$. All participants had normal or corrected-to-normal vision and a mean handedness score (Oldfield, 1971) of +95 (range $=50$ to $+100, S D=15)$ and received payment for their participation ( $£ 10$ plus a variable bonus depending on individual performance; see procedure).

\section{Procedure}

A first practice block of 10 trials was used to familiarize participants with the force-sensitive response keys (see below). In a second practice block consisting of 32 trials, the precuing task was introduced. This was followed by 15 experimental blocks of 64 trials each. Blocks were separated by a short rest. Participants were told the meaning of the precues and were instructed to utilize precue information to shorten RT. There were four stimuli associated with four responses. The four response alternatives consisted of an extension or a flexion of the right or the left index finger. Precues and response signals were designed to be highly compatible with these response alternatives (Figure 1).

The temporal sequence of events is shown in Figure 1. Each trial started with the presentation of the fixation cross $\left(0.2^{\circ} \times 0.2^{\circ}\right.$ visual angle $)$ in the center of the computer screen. After $800 \mathrm{~ms}$, the precue $\left(0.8^{\circ} \times 0.8^{\circ}\right.$ visual angle $)$ was added to the fixation cross. After a constant foreperiod of 1,200 ms, the response signal followed the precue. Participants were asked to keep their eyes on the fixation cross, not to blink as long as it was visible, and to perform the required response as quickly as possible while avoiding response errors. In case of a wrong response, feedback was provided on the screen immediately after response onset. One of the following messages was displayed: (a) "Incorrect" if a wrong response alternative was used or if the participant responded with both hands, (b) "Too fast" if $\mathrm{RT}<100 \mathrm{~ms}$, (c) "Too slow" if there was no response within $1,000 \mathrm{~ms}$. Feedback information was displayed for 1,000 ms. After presentation of feedback or immediately after response onset in the case of correct responses, a blank interval randomly varying between 1,100 and 1,500 ms was presented after which the next trial started. Total mean RT and percentage of response errors were computed for each block and provided as feedback. To keep task compliance at a high level, participants received additional payment of $£ 0.5$ for each block in which responses were faster as compared to the previous block while error rate did not exceed $5 \%$.

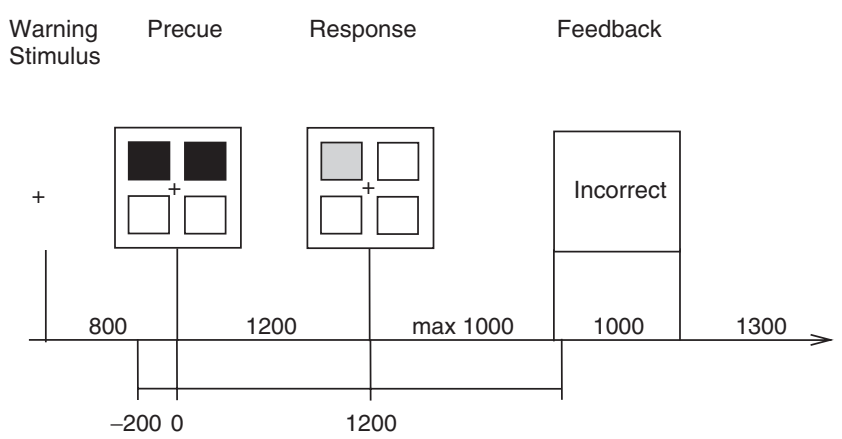

Figure 1. The temporal sequence of events in a single trial. 


\section{Stimuli and Precues}

Ambient light was kept at a low level. A constant viewing distance of $1 \mathrm{~m}$ was introduced. The precues consisted of four small white open squares $\left(0.3^{\circ} \times 0.3^{\circ}\right)$ presented on a black background. The centers of the squares were placed on the corners of an imaginary square $\left(0.8^{\circ} \times 0.8^{\circ}\right)$. Advance information about response hand and about movement direction was provided by the filling pattern (in red) of the squares. The response signal consisted of the same square arrangement as the precue except that a single square filled in green indicated the response.

There were four precue categories. The hand precue provided advance information about the response finger (left vs. right index finger) but no information about movement direction. If the left (right) finger was prespecified, the two left (right) squares were filled red while the two right (left) squares were empty. The direction precue specified movement direction but not the response finger. Two red-filled upper (lower) squares gave advance information about finger extension (flexion). The ambiguous precues reduced the number of response alternatives without prespecifying movement parameters. Here a red filled upper (lower) left square and a red filled lower (upper) right square gave advance information about left finger extension (flexion) or right finger flexion (extension). The noninformative precue consisted of the four unfilled white squares. There were 16 trial types that resulted from the factorial combination of precue category by response alternative. All trial types were randomized within each experimental block. A session included 60 replications of each trial type.

\section{Response Keys}

Force-sensitive keys (cf. Leuthold et al., 1996) were used to register hand responses as soon as force output exceeded a criterion of $0.5 \mathrm{~N}$ from the baseline force level. The force keys were mounted about $35 \mathrm{~cm}$ apart on a board aligned to the body's midline. For flexions and extensions, participants had to move the index fingers down and up, respectively.

\section{Electrophysiological Recordings}

Using a BIOSEMI Active-Two amplifier system, electroencephalographic (EEG) activity was continuously recorded from 70 $\mathrm{Ag} / \mathrm{AgCl}$ electrodes over midline electrodes $\mathrm{FPz}, \mathrm{AFz}, \mathrm{Fz}, \mathrm{FCz}$, $\mathrm{Cz}, \mathrm{CPz}, \mathrm{Pz}, \mathrm{POz}, \mathrm{Oz}$, and $\mathrm{Iz}$, over the left hemisphere from electrodes IO1, Fp1, AF3, AF7, F1, F3, F5, F7, F9, FC1, FC3, FC5, FT7, C1, C3, C5, M1, T7, CP1, CP3, CP5, TP7, P1, P3, P5, $\mathrm{P} 7, \mathrm{P} 9, \mathrm{PO} 3, \mathrm{PO} 7$, and $\mathrm{O} 1$, and from the homolog electrodes over the right hemisphere. Horizontal EOG (hEOG) waveforms were calculated off-line as follows: $h E O G(t)=F 9(t)$ minus $\mathrm{F} 10(\mathrm{t})$. Two additional electrodes (Common Mode Sense [CMS] active electrode and Driven Right Leg [DRL] passive electrode were used as reference and ground electrodes, respectively; cf. http://www.biosemi/faq/cms\&drl.htm). EEG and EOG recordings were sampled at $256 \mathrm{~Hz}$. Off-line, trials containing blinks were corrected using a dipole approach (BESA 2000) and EEG activity was rereferenced to linked mastoids. The analysis epoch for the stimulus-locked data started $200 \mathrm{~ms}$ prior to precue onset and lasted for a total duration of 2,500 ms. For response-locked analyses, epochs were cut starting 1,400 ms before the response and lasting for 2,500 ms. EEG and EOG activity was filtered (bandpass $0.01-10 \mathrm{~Hz}, 6 \mathrm{db} / \mathrm{oct}$ ) and averaged time-locked to precue onset (S-locked data) or to response onset (R-locked data).
In addition, for each participant and each experimental condition, the ERP at recording sites ipsilateral to the response hand was subtracted from the ERP at homologous contralateral recording sites. The resulting difference waveform for each sitepair (e.g., C3/C4) was averaged across hands separately to eliminate any ERP activity unrelated to hand-specific motor activation (cf. Coles, 1989). The term LRP will be exclusively used to describe activity at the $\mathrm{C} 3 / \mathrm{C} 4$ site and the term L-ERP to refer to lateralized ERP activity as calculated in the same way as the LRP for all electrode site-pairs.

\section{Data Analysis}

Only trials with a correct response, with RT between $150 \mathrm{~ms}$ and $1,000 \mathrm{~ms}$, and without EEG or EOG artifacts were included in RT and EEG data analysis. All signals were averaged separately for experimental conditions. Mean foreperiod CNV amplitude and LRP/L-ERP amplitude were analyzed in a 200-ms interval immediately preceding response signal onset using a 200-ms baseline before precue onset. Statistical analyses were performed by means of Huynh-Feldt corrected repeated-measures analyses of variance (ANOVA). For RT and error rate analysis, the within-subject variables were precue category (noninformative precue, direction precue, hand precue, and ambiguous precue), movement direction (extension or flexion), and hand (left or right). For foreperiod L-ERP/LRP amplitude and foreperiod $\mathrm{CNV}$, the data were pooled over factors direction and hand. For post hoc comparisons, the level of significance was Bonferroni adjusted with the alpha level per measure set at $p=.05$.

LRP onsets were measured and analyzed by applying the jackknife-based procedure suggested by Miller, Patterson, and Ulrich (1998) and Ulrich and Miller (2001). Twelve different grand average LRPs for each of the experimental conditions were computed by omitting from each grand average the ERP data of another participant. LRP onsets were determined in the waveform of each grand average.

S-LRP onsets were measured relative to a 200 -ms preresponse signal baseline at the point in time when LRP amplitude reached $30 \%, 50 \%$, or $70 \%$ of maximal LRP amplitude in that specific condition. Effects in the LRP-R interval were obtained using the same relative LRP onset criteria with waveforms referred to a 200-ms baseline starting $900 \mathrm{~ms}$ before the response. An additional analysis of the LRP onset latencies was performed using the regression-based technique (Mordkoff \& Gianaros, 2000; Schwarzenau, Falkenstein, Hoormann, \& Hohnsbein, 1998) in combination with the jackknife-based approach (Ulrich $\&$ Miller, 2001). Following the suggestions of Mordkoff and Gianaros (2000), we employed the so-called $\mathrm{SS} 1_{\mathrm{DF}}$ regression method. This involved fitting of two intersecting lines to each LRP waveform. The pre-onset line was defined as a straight line with a zero slope. It started at $-400 \mathrm{~ms}$ relative to responsesignal onset (S2) for the stimulus-locked LRPs and $-900 \mathrm{~ms}$ relative to response onset for the response-locked LRPs. The post-onset line ended at the LRP peak and was fitted to the LRP data with a linear least square procedure. The time of intersection of the pre-onset line with the post-onset line was taken as LRP onset.

LRP onset measures were submitted to ANOVA with $F$ values corrected as follows: $F_{\mathrm{C}}=F /(\mathrm{n}-1)^{2}$, where $F_{\mathrm{C}}$ denotes the corrected $F$ value and $n$ the number of participants (cf. Ulrich $\&$ Miller, 2001). For all post hoc comparisons, the level of significance was Bonferroni adjusted with the alpha level per measure set at $p=.05$. 
Results

\section{Reaction Time and Error Rate}

Figure 2 provides mean RT and percentage of choice errors. All trials with missing or too slow responses $(0.2 \%)$ and too fast responses $(0.3 \%)$ were discarded from the analysis. A three-way ANOVA of choice error rate including the variables precue category, hand, and movement direction yielded a main effect of hand, $F(1,11)=7.7, p<.05$, due to more errors for left-hand $(2.8 \%)$ than right-hand responses $(1.8 \%)$. No other effects were significant.

The analogous analysis of RT yielded a main effect of precue category, $F(3,33)=24.5, p<.001, \varepsilon=.78$. Responses were slower for the noninformative precue $(344 \mathrm{~ms})$ compared to the direction precue condition $(319 \mathrm{~ms})$ and the hand precue condition ( $324 \mathrm{~ms}$ ) as well as compared to the ambiguous precue (323 ms). The latter three precue conditions did not differ from each other in RT. Responses were faster for extension (322 ms) than flexion movements $(333 \mathrm{~ms}), F(1,11)=8.6, p<.05$. Also responses with the right hand $(324 \mathrm{~ms})$ were faster than with the left hand $(331 \mathrm{~ms}), F(1,11)=9.7, p<.01$. No interactions between experimental variables were significant.

\section{Foreperiod L-ERP/LRP Amplitude}

As can be seen in Figure 3, the foreperiod L-ERP/LRP deviated from zero about $200 \mathrm{~ms}$ after precue onset if the hand was precued. Because this hand precue condition contained lateralized stimulus information, this LRP effect most probably reflects transient contributions of lateralized sensory activity that is of largest amplitude over electrodes PO7/PO8 (cf. Figure 3). Later on in the foreperiod interval, as expected, the LRP deviated from baseline only when information about the response hand was provided in advance, indicating preparation of the forthcoming response hand. The ANOVA of L-ERP amplitudes, measured in a 200-ms interval immediately preceding the

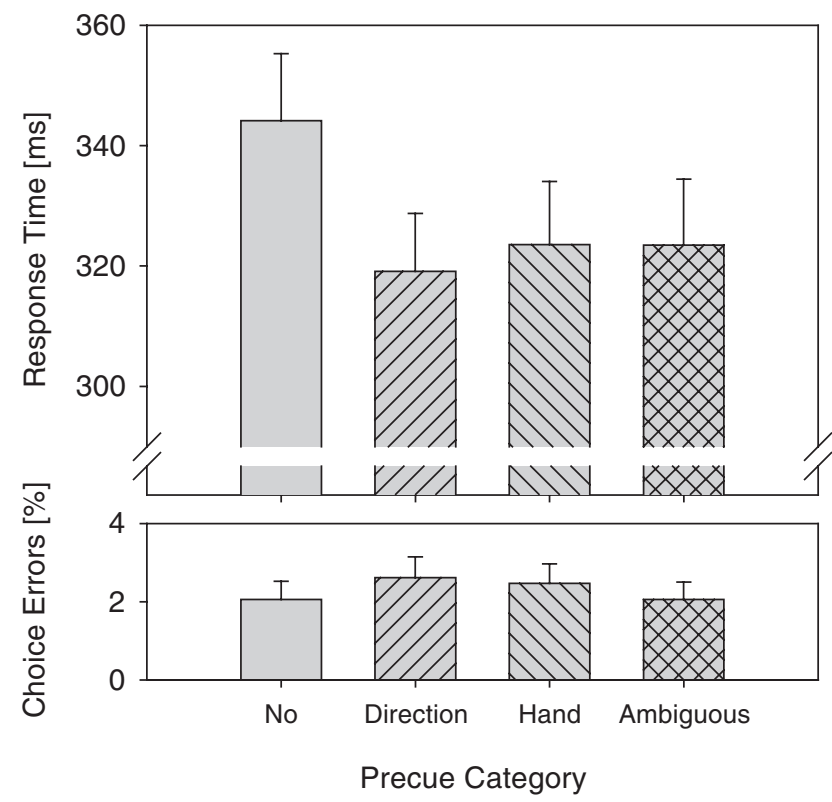

Figure 2. Mean response times (top) and error rates (bottom) for the four precue categories noninformative precue (No), direction precue, hand precue, and ambiguous precue. Error bars represent standard errors for the individual means.

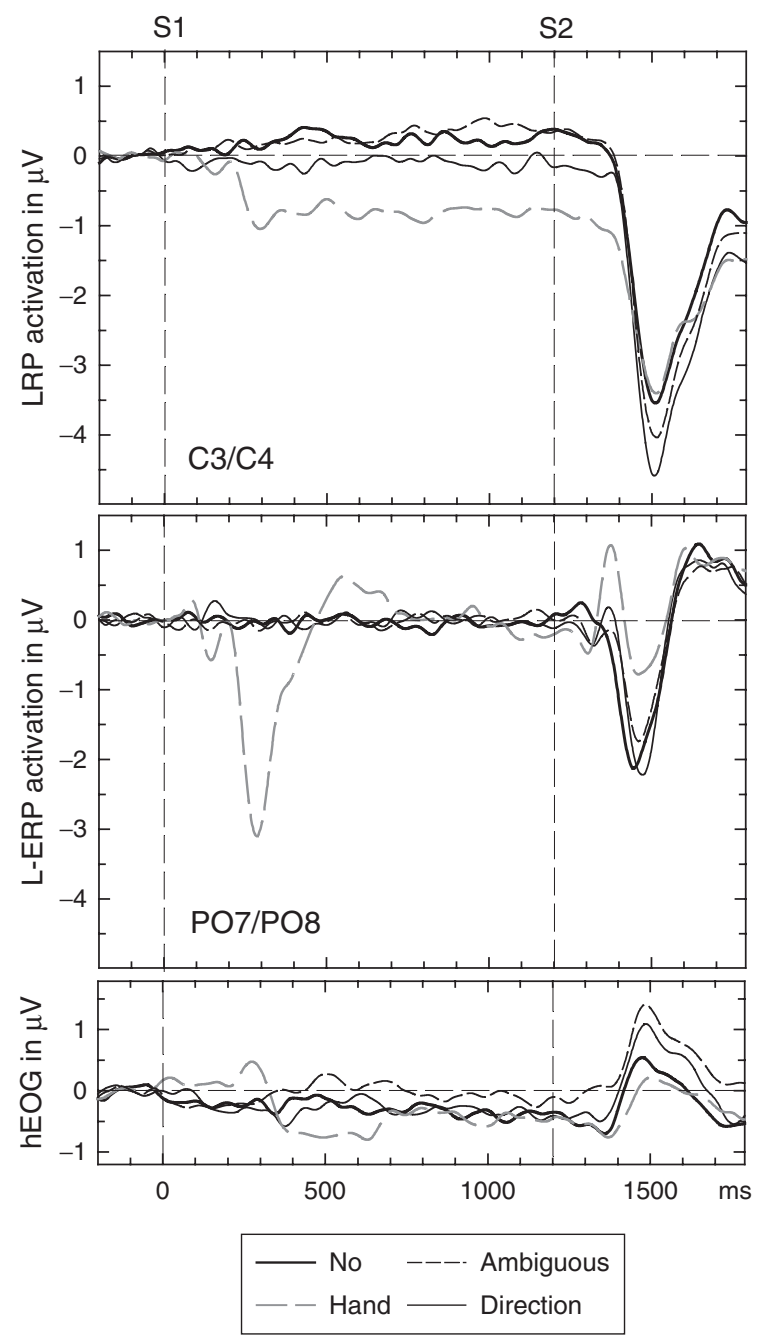

Figure 3. Stimulus-locked lateralized ERP waveforms for precue categories noninformative precue (No), direction precue, hand precue, and ambiguous precue at electrodes $\mathrm{C} 3 / \mathrm{C} 4$ and $\mathrm{PO} 7 / \mathrm{PO} 8$ and for the hEOG. S1 and S2 refer to precue and response signal onset, respectively.

response signal, included the variables precue category and electrode (30 levels). This analysis revealed a main effect of electrode, $F(29,319)=2.0, p<.01, \varepsilon=.74$, and an effect of precue category, $F(3,33)=8.2, p<.001, \varepsilon=.90$, which was due to the following ordering of L-ERP amplitudes: hand precue $(-0.38 \mu \mathrm{V})<$ direction precue $(-0.04 \mu \mathrm{V})=$ ambiguous precue $(0.12 \mu \mathrm{V})=$ noninformative precue $(0.07 \mu \mathrm{V})$. No other effects approached significance.

The analysis of LRP amplitude (at C3/C4) showed also a significant effect of precue category, $F(3,33)=10.2, p<.001$, $\varepsilon=.84$, with LRP amplitudes ordered as follows: hand precue $(-0.77 \mu \mathrm{V})<$ direction precue $(-0.05 \mu \mathrm{V})=$ ambiguous precue $(0.41 \mu \mathrm{V})=$ noninformative precue $(0.13 \mu \mathrm{V})$. A $t$ test revealed that LRP amplitude differed significantly from zero only if hand was known in advance, $t(11)=-3.1, p<.01$.

\section{LRP Onsets}

As seen in Figure 3 and revealed by the foreperiod LRP amplitude analyses, a significant LRP already emerges before response signal onset in the hand precue condition, making the interpretation of any onset measure for this condition inherently 


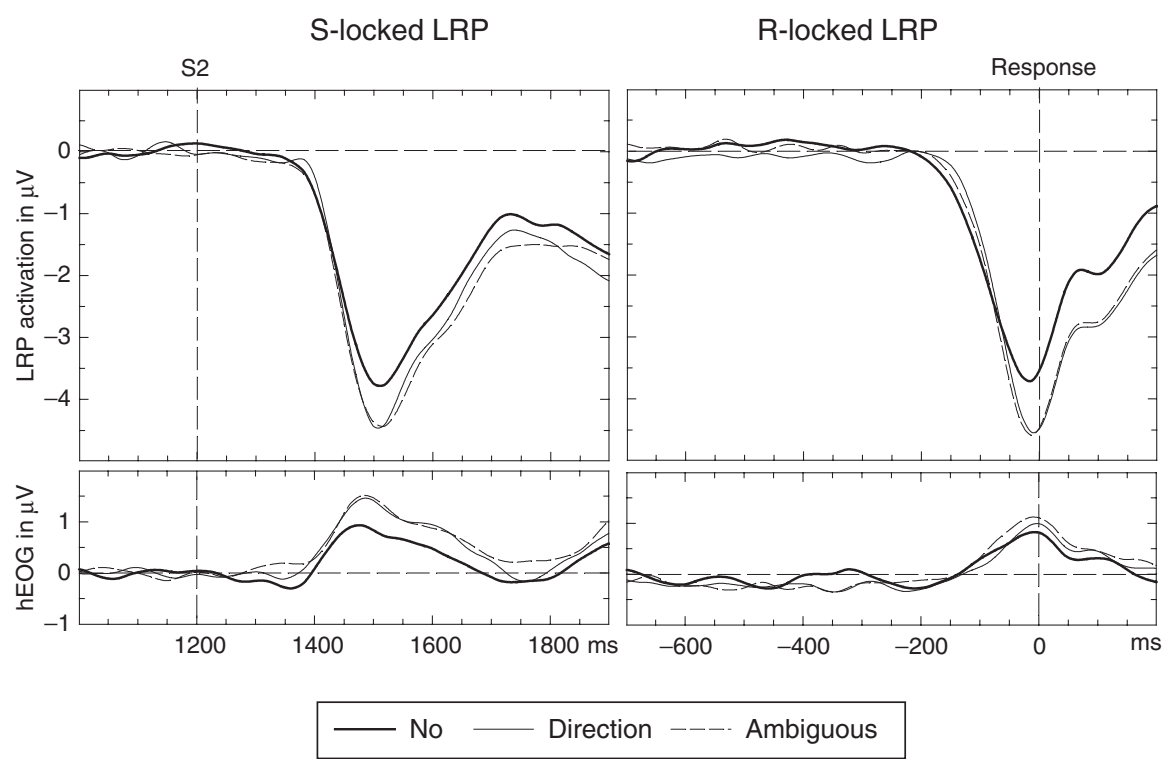

Figure 4. Stimulus-locked LRPs (left panel) and response-locked LRPs (right panel) and the corresponding hEOG waveforms superimposed for precue categories noninformative precue (No), direction precue, and ambiguous precue. S2 indicates response signal onset.

difficult. Therefore, we decided to analyze the LRP onsets only for the noninformative, direction, and ambiguous precue conditions. Figure 4 shows the stimulus-locked LRPs (left panel) and the response-locked LRPs (right panel) for these three precue categories. Moreover, as can be seen in Figure 3, lateral stimulus presentation clearly produced a lateralized activity with a maximum at PO7/PO8 electrode sites. This activity projects to centrolateral recording sites, as indicated by an contribution of about $25 \%$ of maximal PO7/PO8 amplitude to the activity measured at $\mathrm{C} 3 / \mathrm{C} 4$ electrode sites when estimated from the dipole source model (see the Appendix). This value roughly agrees with a sensory-induced contribution to the amplitudes measured at centrolateral electrode sites of about $34 \%$ as reported by Oostenveld, Praamstra, Stegeman, and van Oosterom (2001). As a result, asymmetric sensory activity might affect LRP-onset measures especially when relatively low voltage criteria are applied. As mentioned earlier, we introduced here an alternative approach to deal with this problem and measured in addition onsets in dipole-corrected waveforms, for which influences of sensory activity are largely reduced. This approach is outlined in detail in the Appendix to keep the present section short. Crucially, the onset results obtained for dipole-fitted waveforms replicated the pattern found in the LRPs reported in the next section.

ANOVA results of the S-LRP interval values for the different onset criteria and baselines are shown in Table 1. The duration of the S-LRP interval (values given for the $50 \%$ criterion) did not differ among noninformative $(244 \mathrm{~ms})$, direction $(245 \mathrm{~ms})$, and ambiguous precue conditions ( $238 \mathrm{~ms})$. This finding held true for all LRP-onset criteria.

In contrast to the results for the S-LRP interval, clear precuing effects were found in the LRP-R interval (see Figure 4, right, and Table 2). Motoric processing time (means given for the $50 \%$ criterion) was longer when no advance information about the upcoming movement was provided $(104 \mathrm{~ms})$ as compared to when direction was precued $(80 \mathrm{~ms})$ or ambiguous information was delivered (84 ms).

\section{Foreperiod CNV Amplitude}

The two-way ANOVA of foreperiod CNV amplitude, measured as the mean amplitude in a $200-\mathrm{ms}$ time interval immediately preceding the response signal, included the variables precue category and electrode (68 levels). As shown in the spline maps in the lower part of Figure 5, CNV amplitude was largest at medial central regions, resulting in a significant main effect of electrode, $F(67,737)=19.3, p<.001, \varepsilon=.18$. The CNV amplitude was strongly influenced by precue category, $F(3,33)=13.7, p<.001$, $\varepsilon=1.00$. Post hoc comparison indicated a smaller foreperiod $\mathrm{CNV}$ for the noninformative precue $(-2.1 \mu \mathrm{V})$ than the precue conditions direction $(-4.0 \mu \mathrm{V})$, hand $(-3.2 \mu \mathrm{V})$, and ambiguous precue $(-3.7 \mu \mathrm{V})$. In addition, foreperiod $\mathrm{CNV}$ was more negative if direction rather than hand was precued,

Table 1. F $F_{C}$ Values: ANOVA of the Effects of Precue Category in the S-LRP Interval for the Three Relative Onset Criteria $70 \%$, 50\%, and $30 \%$ and the Regression-Based Method $S S 1_{D F}$

\begin{tabular}{|c|c|c|c|c|c|c|c|c|c|}
\hline & \multirow[b]{2}{*}{$d f$} & \multicolumn{4}{|c|}{ LRP values } & \multicolumn{4}{|c|}{ Dipole corrected values } \\
\hline & & $70 \%$ & $50 \%$ & $30 \%$ & $\mathrm{SS} 1_{\mathrm{DF}}$ & $70 \%$ & $50 \%$ & $30 \%$ & $\mathrm{SS}_{\mathrm{DF}}$ \\
\hline Overall & 2,22 & 0.9 & 0.9 & 1.3 & 1.8 & 0.2 & 0.2 & 0.9 & 0.9 \\
\hline NP vs. DP & 1,11 & 0.0 & 0.0 & 1.4 & 4.1 & 0.3 & 0.8 & 2.2 & 2.6 \\
\hline NP vs. AP & 1,11 & 1.2 & 1.0 & 0.1 & 0.4 & 0.3 & 1.1 & 0.8 & 0.7 \\
\hline
\end{tabular}

NP: noninformative precue, DP: direction precue, AP: ambiguous precue. 
Table 2. $F_{C}$ Values: ANOVA of the Effects of Precue Category in the LRP-R interval for the Three Relative Onset Criteria $70 \%, 50 \%$, and $30 \%$ and the Regression-Based Method $S S 1_{D F}$

\begin{tabular}{|c|c|c|c|c|c|c|c|c|c|}
\hline & \multirow[b]{2}{*}{$d f$} & \multicolumn{4}{|c|}{ LRP values } & \multicolumn{4}{|c|}{ Dipole corrected values } \\
\hline & & $70 \%$ & $50 \%$ & $30 \%$ & $\mathrm{SS} 1_{\mathrm{DF}}$ & $70 \%$ & $50 \%$ & $30 \%$ & $S S 1_{D F}$ \\
\hline Overall & 2,22 & $5.3^{*}$ & $11.3^{* * *}$ & $4.8^{*}$ & $21.4^{* * * * *}$ & $3.4^{*}$ & $5.9^{* *}$ & $6.2^{* *}$ & $7.1^{* * *}$ \\
\hline NP vs. DP & 1,11 & $8.8^{*}$ & $21.6^{* * *}$ & $6.1^{(*)}$ & $32.0^{* * * * * *}$ & $16.0^{* * *}$ & $7.9 *$ & $10.6^{*}$ & $7.7^{*}$ \\
\hline NP vs. AP & 1,11 & $5.3^{(*)}$ & $9.6^{*}$ & $5.1^{(*)}$ & $16.1^{* * * * * *}$ & 2.9 & $5.5^{(*)}$ & $8.0 *$ & $7.9 *$ \\
\hline
\end{tabular}

NP: noninformative precue, DP: direction precue, AP: ambiguous precue.

${ }^{(*)} p<.1 ; * p<.05 ; * * p<.01 ; * * * p<.001$.

$F(1,11)=17.4, p<.01$. Precue category interacted with electrode, $F(201,2211)=8.2, p<.001, \varepsilon=.13$, due to strongest precuing effects over central electrodes.

To make the present study comparable to other precuing studies, we also analyzed the $\mathrm{CNV}$ amplitude at the $\mathrm{Cz}$ electrode alone (see Figure 5, upper part). The results were equivalent to the ones obtained in the above analysis including all electrodes. A clear precuing effect was found in CNV amplitude, $F(3,33)=25.3, p<.001, \varepsilon=1.00$. As before, post hoc comparison revealed a reliably smaller foreperiod $\mathrm{CNV}$ for the noninformative precue $(-5.7 \mu \mathrm{V})$ as compared to the direction precue $(-10.7 \mu \mathrm{V})$, the hand precue $(-9.3 \mu \mathrm{V})$, and the ambiguous precue $(-10.3 \mu \mathrm{V})$ as well as a reliable amplitude difference between direction and hand precue conditions, $F \mathrm{~s}(1$, 11) $>7.5, p s<.05$.

\section{Lateral ERP Activity over MI}

In this analysis step, we investigated the hypothesis of multiple motor preparation by applying the same analysis logic as in one

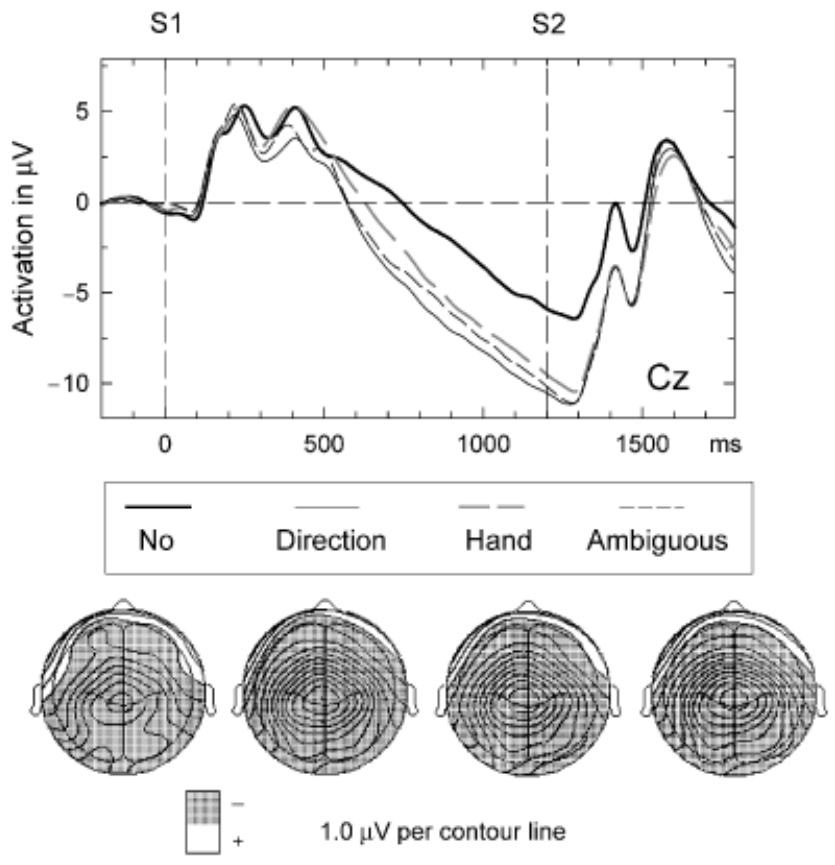

Figure 5. Top: Stimulus-locked ERPs at the Cz electrode superimposed for precue categories noninformative precue (No), direction precue, hand precue, and ambiguous precue. $\mathrm{S} 1$ and $\mathrm{S} 2$ indicate precue and response signal, respectively. Bottom: Spline maps for the ERP activation averaged across a time interval from -200 to $0 \mathrm{~ms}$ relative to S2 onset. Negativity is printed shaded and positivity printed unshaded. of our previous studies (Leuthold \& Jentzsch, 2001) where we found a parallel preparation of both response hands when advance information about direction was provided. The idea in the present study was that preparation of both response hands in the direction and ambiguous precue conditions should show up in an equally large activity ipsi- and contralateral to the responding hand. Most crucially, the ipsilateral activity should be larger as compared to a condition where the response hand is known in advance (hand precue).

Foreperiod CNV amplitudes were analyzed at electrodes C3 and C4 only (see Figure 6), because at these electrodes preparatory motor activity was largest. A priori contrasts testing these predictions revealed a larger foreperiod CNV amplitudes at the electrode site ipsilateral to the responding hand for the direction precue $(-7.1 \mu \mathrm{V})$ than the hand precue $(-5.5 \mu \mathrm{V}), F(1$, $11)=10.4, p<.01$, and the ambiguous precue $(-6.8 \mu \mathrm{V})$ than the hand precue, $F(1,11)=4.9, p<.05$. In contrast, $\mathrm{CNV}$ amplitude at the electrode site contralateral to the responding hand was not differentially influenced by precue condition, $M$ (direction precue, hand precue, and ambiguous precue $)=-7.3,-6.5$, and $-6.6 \mu \mathrm{V}), F(2,22)=1.4, p=.26, \varepsilon=.77$.

Moreover, it was only the hand precue condition that showed a response-related lateralization (comparison ipsi vs. contra) of the foreperiod $\mathrm{CNV}, F(1,11)=15.5, p<.01$, reflecting the one obtained in the LRP, whereas the direction and ambiguous precue conditions showed no such lateralization effect, $F_{\mathrm{S}}<1.6$.

\section{RT Bin Analysis}

The hypothesis of a strategic influence on the results obtained for the ambiguous precue condition, namely, a trial-by-trial preparation for just one of the two alternatives, was tested by analyzing ERP data categorized according to response latency. That is, for each participant and each condition separately, the RT distribution was divided equally into three RT bins of fast, medium, and slow responses. In the following, only effects of the variable RT bin and its interaction with other variables will be reported to avoid redundancy. Data were pooled across the variables direction and hand to increase the number of trials available for each RT bin.

Reaction time. The ANOVA included the variables precue category and RT bin (fast, medium, slow). This analysis yielded a main effect of RT bin, $M$ (fast, medium, slow) $=266,320$, and $387 \mathrm{~ms}, \quad F(2,22)=127.3, p<.001, \varepsilon=.66$, which was not significantly modulated by precue category, $F(6,66)=2.2$, $p>.08, \varepsilon=.71$.

Foreperiod L-ERP/LRP amplitude. Figure 7 depicts the RT bin effect in the foreperiod LRP waveforms for the four precue conditions (top to bottom). No main effect of RT bin, $F_{\mathrm{S}}<2.0$, 

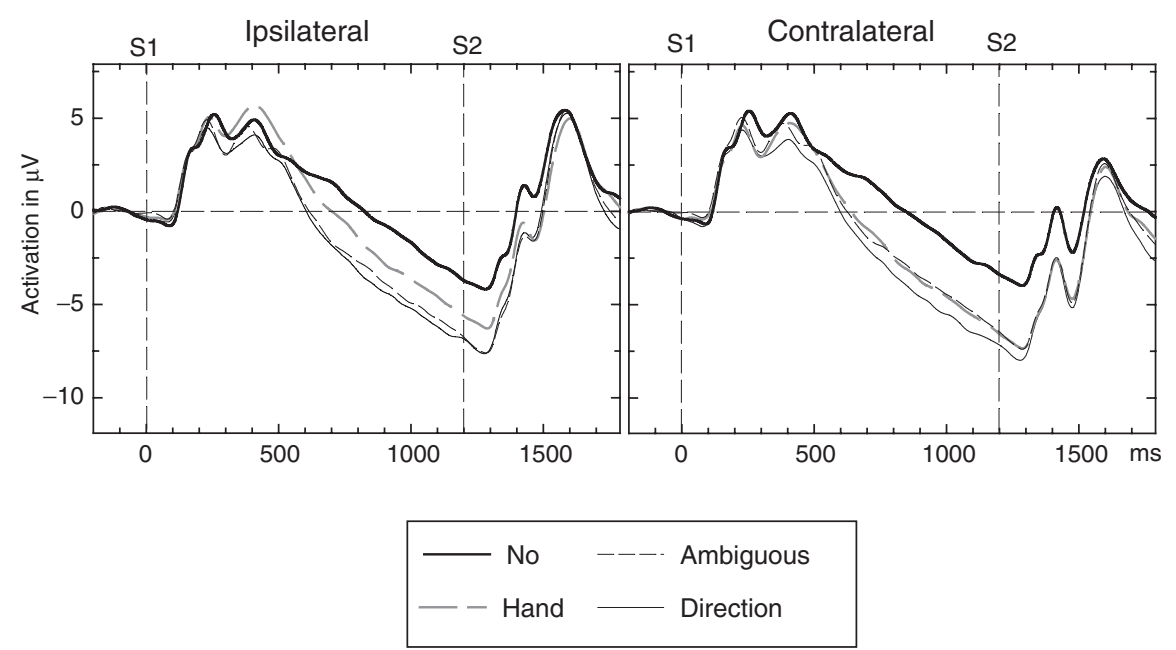

Figure 6. Stimulus-locked ERPs at the central electrodes (C3 and C4) ipsilateral (left panel) and contralateral (right panel) to the responding hand, superimposed for precue categories noninformative precue (No), direction precue, hand precue, and ambiguous precue. S1 and S2 indicate precue and response signal, respectively.

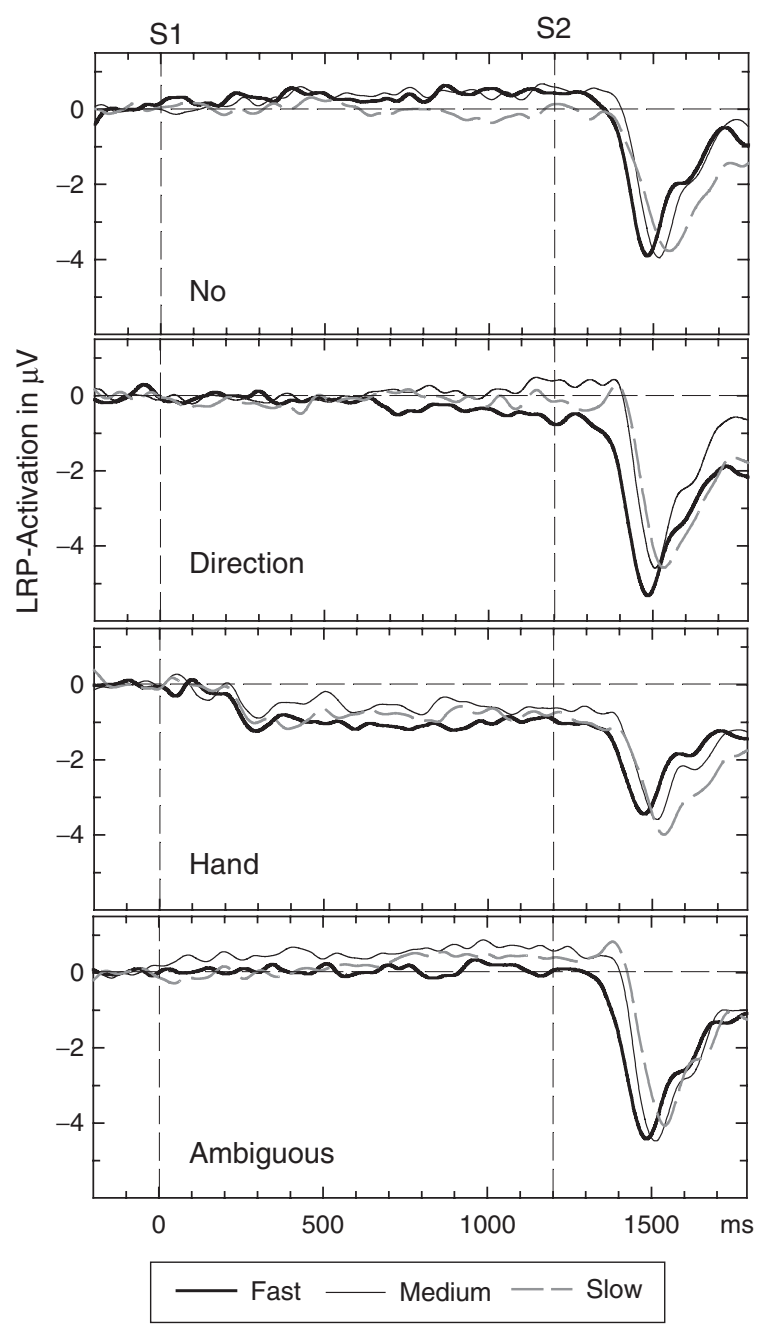

Figure 7. Stimulus-locked LRPs for precue categories delivering No information (top panel), partial information about response direction (second panel), about response hand (third panel), and ambiguous information (bottom panel). The waveforms for fast, medium, and slow responses are superimposed. S1 and S2 indicate precue and response signal, respectively. $p \mathrm{~s}>.16$, nor any significant interactions with $\mathrm{RT}$ bin were found in the analysis of foreperiod L-ERP/LRP amplitudes, all $F_{\mathrm{S}}<1.1, p \mathrm{~s}>.3$.

LRP onset. For the reasons provided earlier, only the data for the noninformative, direction, and ambiguous precues were included in the RT bin analysis of LRP onsets; the ANOVA results for S-LRP onsets are shown in Table 3 (see also Figure 8, left-hand side) and those for the LRP-R onsets in Table 4 (see also Figure 8, right-hand side). The duration of the S-LRP interval (values given for the $50 \%$ criterion) was influenced by RT bin, being shortest for the fast RT bin ( $218 \mathrm{~ms})$, intermediate for the medium RT bin (252 ms), and longest for the slow RT bin $(266 \mathrm{~ms})$. Note that this effect was present irrespective of the specific criterion applied and, most importantly, it was also not modulated by precue category.

The duration of the LRP-R interval (values given for the $50 \%$ criterion) was marginally influenced by speed with equally long intervals for the fast and medium bin (78 and $81 \mathrm{~ms}$ ), but a prolonged LRP-R interval for the slow bin $(110 \mathrm{~ms})$. This effect was significant for the three relative onset criteria and not modulated by precue category.

\section{Discussion}

The aim of the present study was to investigate the nature of response time benefits that are found for ambiguous precue conditions. Although previous evidence suggests a motoric locus of the effect (Osman et al., 1995), no clear electrophysiological evidence has been put forward in explaining why a precue condition providing no advance parameter-specific information should speed up motoric processing time. Such a motoric locus would challenge the standard view according to which ambiguous precues shorten the premotoric portion of RT by reducing the number of S-R alternatives (e.g., Goodman \& Kelso, 1980). One primary aim of the present experiment therefore was to examine the premotoric or motoric locus of the RT benefit in the ambiguous condition and to test the hypothesis proposed earlier that multiple response preparation might occur under such conditions (e.g., Larish \& Frekany, 1985) using a chronopsy- 
Table 3. $F_{C}$ Values: ANOVA of the Effects of Speed in the S-LRP Interval for the Three Relative Onset Criteria 70\%, 50\%, and 30\% and the Regression-Based Method $S S 1_{D F}$

\begin{tabular}{lccccc}
\hline \hline & $d f$ & $70 \%$ & $50^{*}$ & $30^{*}$ & $\mathrm{SS} 1_{\mathrm{DF}}$ \\
\hline Overall & 2,22 & $37.6^{* * * *}$ & $39.5^{* * * *}$ & $31.3^{* * * *}$ & $29.2^{* * * *}$ \\
Fast vs. medium & 1,11 & $27.6^{* * *}$ & $32.2^{* * * * *}$ & $32.8^{* * * * * *}$ & $24.5^{* * *}$ \\
Fast vs. slow & 1,11 & $51.1^{* * * *}$ & $53.8^{* * * *}$ & $39.1^{* * * *}$ & $47.4^{* * * *}$ \\
Medium vs. slow & 1,11 & $23.0^{* * *}$ & $20.1^{* * *}$ & $11.0^{*}$ & 2.4 \\
\hline \hline
\end{tabular}

${ }^{*} p<.05 ; * * p<.01 ; * * * p<.001$.

chophysiological approach (cf. Van der Molen, Bashore, Halliday, \& Callaway, 1991).

\section{Ambiguous-Precue Benefits: Motoric or Premotoric Locus?}

In a response precuing task that involved flexion or extension movements with the left or right index finger, a spatially compatible precue conveyed information about response side, information about direction, ambiguous information that reduced the number of S-R alternatives to two, or no information at all. Behavioral and electrophysiological findings indicated the utilization of precue information for advance preparation. Thus, responses were faster for partial precues than the no-information precue. This precuing effect in RT replicates earlier reports that showed a shortening of $\mathrm{S}-\mathrm{R}$ processing time with advance information about movement parameters hand and direction (e.g., Leuthold et al., 1996). In addition, the ambiguous precue shortened RT by the same amount as both partial precues, that is, no differential precuing effect was obtained. Thus, present RT findings resemble those reported by Osman et al. (1995) for a precuing task that included response dimensions hand and finger. Previously, Goodman and Kelso (1980) took the finding of a nondifferential precuing effect obtained with spatially compatible S-R mappings in combination with an identical RT reduction for ambiguous and partial precues as particularly strong evidence for their S-R translation hypothesis and against Rosenbaum's $(1980,1983)$ hypothesis of advance motor progra- mming. Here, we distinguished between these two positions by analyzing stimulus- and response-locked LRP onsets.

Most important, the LRP onset analyses revealed a clear and exclusive motoric origin of the RT benefits for both the direction precue and the ambiguous precue condition compared to the noninformative precue. As in a previous LRP study that used a spatially compatible S-R mapping (Leuthold et al., 1996), a precuing effect showed up as a reduction in the LRP-R interval in the direction precue condition. A comparable reduction of the LRP-R interval for the ambiguous precue strongly suggests that the ambiguous precue shortened motoric processing time. This finding corresponds to a similar motoric effect of the ambiguous precue in the study of Osman et al. (1995); however, in their study, partial and ambiguous precues shortened premotoric processing time as well. Presumably, the present exclusive motoric precuing effect is due to the enhanced spatial S-R compatibility in the present study, because a horizontal mapping of stimulus side to response side was used instead of the less direct vertical S-R arrangement in Osman et al.'s study. According to our results, then, specification of movement direction and hand shortened mainly motoric but not reliably premotoric processes. This conjecture challenges the prevailing assumption according to which specification costs in precuing tasks using spatially compatible S-R mappings and ambiguous precue conditions reflect a genuine effect at premotor stages (e.g., Goodman \& Kelso, 1980).

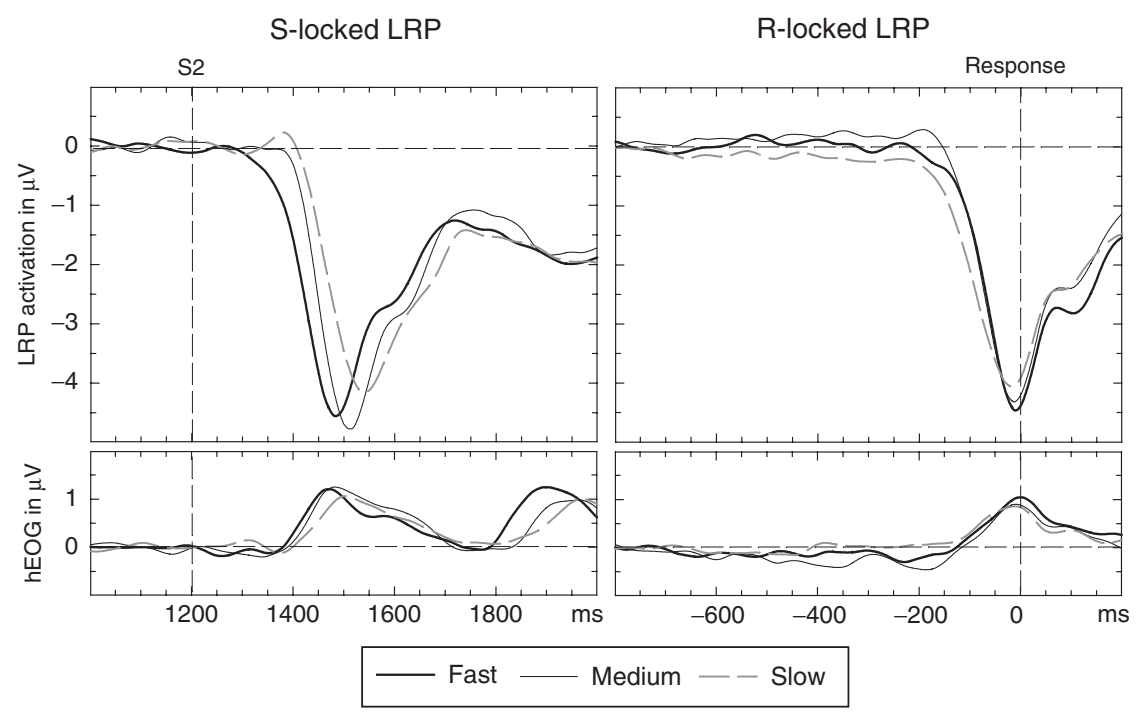

Figure 8. Stimulus-locked LRPs (left panel) and response-locked LRPs (right panel) and the corresponding hEOG waveforms (collapsed across conditions no precue, direction precue, and ambiguous precue) superimposed for the response speed categories (fast, medium, and slow). S2 indicates response signal onset. 
Table 4. $F_{C}$ Values: ANOVA of the Effects of Speed in the LRP-R Interval for the Three Relative Onset Criteria $70 \%, 50 \%$, and $30 \%$ and the Regression-Based Method $S S 1_{D F}$

\begin{tabular}{lccccc}
\hline \hline & $d f$ & $70 \%$ & $50 \%$ & $30 \%$ & $\mathrm{SS} 1_{\mathrm{DF}}$ \\
\hline Overall & 2,22 & $8.0^{* * *}$ & $13.3^{* * *}$ & $10.0^{* * *}$ & $5.2^{*}$ \\
Fast vs. medium & 1,11 & 0.5 & 0.5 & 0.0 & 0.0 \\
Fast vs. slow & 1,11 & $9.5^{*}$ & $16.1^{* *}$ & $12.3^{*}$ & 5.1 \\
Medium vs. slow & 1,11 & $10.8^{*}$ & $15.8^{* *}$ & $14.8^{* *}$ & $7.3^{* *}$ \\
\hline \hline
\end{tabular}

${ }^{* *} p<.1 ; * p<.05 ; * * *<<.01$

Of course, proponents of the S-R translation view might still argue that there are problems with the measurement of LRP onset in the present study due to the possible overlap of asymmetric sensory activity. Thus, the absence of a precuing effect in the S-LRP interval and its presence in the LRP-R interval might be attributed to the fact that actually LRP onset measures were distorted by overlapping asymmetric sensory activity. We will argue in the following why this sensory overlap explanation cannot account for present findings.

First, it should be noticed that asymmetric sensory activity maximal over occipitoparietal sites was attenuated by about $25 \%$ at electrodes located over lateral motor cortices. Thus, only when LRP onsets are determined with a relatively low threshold might it be possible for this sensory activity to mimic the onset of motor activation. In contrast, we found our LRP onset measures to be very robust as concerns the onset criteria used. Secondly, the analysis of speed classified LRP waveforms provides additional support against the sensory overlap account. If LRP onsets partly reflect the onset of lateralized sensory activity, S-LRP onsets should remain relatively constant despite strong RT variations. This is because the response signal elicits sensory activity with a constant onset and peak irrespective of RT. The RT bin analysis, however, provided clear evidence for a speedrelated modulation of the S-LRP interval, that is, premotoric processing time as indicated by S-LRP onsets became increasingly shorter with decreasing response latency. On the other hand, because the time-course of motoric activity varies with RT whereas asymmetric sensory activity is invariant, it follows that a speed-related effect on the LRP-R interval should be observed if overlapping sensory activity is mimicking motor activity. However, the LRP-R interval was only prolonged for the slowest RT bin but not for fast and medium RT bins, despite the fact that RTs indicated a difference of about $54 \mathrm{~ms}$ between these two speed categories. Finally, and most importantly, we used a dipole source modeling approach to reduce as much as possible the influence of asymmetric sensory activity on lateralized motor activity. When onset measurement was conducted in these dipole-corrected LRP waveforms, the pattern of results replicated the findings of the earlier analysis performed in uncorrected waveforms. It is for these reasons that we rule out the sensoryoverlap account as a sensible explanation of the present LRP onset effects.

A final word of caution concerning the inference of an exclusive motoric locus of the precuing effect is nevertheless needed because such an inference critically depends on the specific temporal arrangement of premotoric and motoric processing stages. Thus, Osman et al. (1995) and Leuthold et al. (1996) pointed out that the interpretation of LRP-R latency effects becomes less clear when premotoric and motoric stages overlap in time. According to such an overlap hypothesis, one might assume that the response in the noninformative precue condition is determined in two successive steps within the response selection stage, for example, because the horizontal S-R dimension is more salient than the vertical one. For the present experiment, then, the side of the response signal (left or right) might be processed first (step 1), providing information about response hand. Only subsequently (step 2 ) is the vertical position processed (top or bottom), providing information about movement direction. Consequently, hand preparation and thus the LRP will start as soon as step 1 has been completed, whereas preparation of movement direction must await completion of step 2. Now it is reasonable to assume that for direction precues, only the first processing step (hand selection) is required after presentation of the response signal, because the precue already provided information about movement direction. The same scenario should hold true for the ambiguous precue, because information about the response side is sufficient to trigger the appropriate response. Consequently, the S-LRP will begin at the same time point for direction, ambiguous, and noninformative precue conditions. However, in the noninformative precue condition, the LRP begins immediately after the hand has been determined in step 1 processing. Critically, extra time is needed for the processing of the vertical position in step 2 (movement direction) before the response can be executed. Thus, this second step of nonmotoric response selection prolongs the LRP-R interval due to asynchronous output from the premotoric selection stage to the motoric stage. As a result, the LRP-R interval will be longer for the noninformative precue than the direction precue and the ambiguous precue.

Although such an overlap hypothesis cannot be completely ruled out on the basis of present LRP findings, we think that such an account is not very plausible given previous reports of a motoric locus of the precuing effect in studies where symbolic stimuli were used. That is, Leuthold et al. (1996,Experiment 2) and Müller-Gethmann et al. (2000) revealed a clear precue effect on the response-locked LRP interval with both symbolic precues and response signals. Importantly, one-dimensional symbolic response signals (e.g., digits or letters assigned to response alternatives) provide much less opportunity for partial S-R translation output to the motor stage compared to twodimensional spatial stimuli (horizontal and vertical dimensions). Thus, the reduction of motoric processing time with information about movement direction is well established (Leuthold et al., 1996; Müller-Gethmann et al., 2000) and in this respect, the present findings for the direction precue condition completely accord with these earlier results.

In addition, there is also evidence suggesting that direction and ambiguous precues influenced preparation at the motoric level before response signal onset, hence, contributing to savings in RT as compared to the noninformative precue condition. Thus, the foreperiod CNV analysis indicated that participants used ambiguous precues to prepare for the forthcoming response before response signal onset. Previous precuing studies using parameters hand and direction (e.g., Ulrich et al., 1998) showed the smallest foreperiod CNV with noninformative precue and increasingly larger CNVs with increases in the amount of advance information. Importantly, if ambiguous precues only reduce the number of $\mathrm{S}-\mathrm{R}$ alternatives, this precue condition should show a foreperiod CNV of a comparable amplitude as for noninformative precues. However, foreperiod CNV amplitude for the ambiguous precue condition, like the CNV obtained for 
hand and direction precues, was larger than for the noninformative precue. This finding is relevant because it indicates that participants used the ambiguous precue condition in a way similar to that used in the partial precue conditions. In the next section, which deals with the mechanisms underlying ambiguousprecue benefits, we will discuss this effect in more detail.

\section{Ambiguous-Precue RT Benefits: Multiple Response Preparation or Strategic Guessing?}

A second major objective was to examine the possible mechanism underlying the precuing effect in ambiguous and direction precue conditions. In particular, we were interested in finding out why an ambiguous condition speeds up motoric processing time. In the following, we will discuss two possible ways by which such a motoric precuing effect might have been induced. On the one hand, it is conceivable that participants actually prepared multiple responses in parallel, as previously suggested (Larish \& Frekany, 1985; Rosenbaum \& Kornblum, 1982). On the other hand, it is also possible that participants adopted some sort of guessing strategy, preparing in full on each trial just one of the two possible ambiguous movements.

Present findings conform with the multiple motor preparation hypothesis, according to which participants prepared the unspecified or ambiguous response alternatives in parallel with direction and ambiguous precues, respectively. As predicted by the multiple motor preparation view, CNV activity over motor cortices contralateral to the responding hand was of equally large amplitude immediately preceding the response signal for conditions direction precue, ambiguous precue, and hand precue. Because only for the hand precue was the responding hand known in advance, this finding suggests the same level of activity in motor areas contralateral to the response hand. Especially important, then, CNV activity over ipsilateral motor areas was larger if movement direction or two ambiguous movements were precued than if hand was known in advance. Note at the same time that the contralateral CNV was found to be larger than the ipsilateral CNV only for the hand precue. This pattern of results can be most easily interpreted by assuming that both response hands were activated to the same level for direction and ambiguous precues. Thus, a multiple preparation of responses was not only found for the direction precue condition as in our earlier study (Leuthold \& Jentzsch, 2001), but for the first time, for an ambiguous precue condition.

It is important to note that present findings cannot be explained by strategic effects on motor preparation, as indicated by the analysis of response latency-classified foreperiod LRP amplitude. Thus, the predicted pattern of correct versus incorrect foreperiod LRP lateralization for fast and slow RT bins in the ambiguous precue condition was clearly absent in the analysis of foreperiod L-ERP/LRP amplitudes. Actually, foreperiod L-
ERP/LRP amplitudes did not change as a function of response speed. Of course, it is always difficult to rule out a hypothesis on the basis of null effects. However, when putting all findings together, the general picture obtained suggests that participants did not engage in a strategy of guessing one of the remaining alternatives when ambiguous or direction information about the forthcoming movement was provided.

These findings together support the notion that two fully prepared responses can be held in short-term motor memory, which is theoretically interesting when considered in the light of suggested capacity limitations within the motor system (Rosenbaum, 1991). Thus, parallel preparation in the case of the direction precue might be possible with the activation of just one motor program, with the parameter response hand being specified at a lower level (cf. Jentzsch \& Leuthold, 2002). However, it is also possible that two independent motor programs are compiled and activated within the two hemispheres (e.g., Cardoso de Oliveira, 2002). For the ambiguous precue condition, the assumption of hand-specific motor programs appears necessary to account for the precuing benefit in RT and the LRP-R interval, because movements differing in all their parameters (e.g., left flexion vs. right extension) cannot be prespecified within a single motor program. Generally, assuming separate motor programs for each hand would have the advantage of explaining the present precuing effect for the direction precue and the ambiguous precue by a common principle. This idea of separate hand-specific motor programs also corresponds with earlier findings of Rosenbaum and Kornblum (1982) who found evidence for a simultaneous preparation of one finger on each hand. Thus, the finding of reliably larger foreperiod $\mathrm{CNV}$ for the direction than the hand precue condition as well as the numerically larger CNV for the ambiguous than the hand precue condition in the present study might reflect the activation of separate motor programs for the left and right hands.

In conclusion, the present experiment provided evidence in support of a motoric locus of response time benefits in ambiguous precue conditions, clearly in contradiction to the suggestion of an exclusively premotoric locus due to the reduction of S-R alternatives. Separation of the data into three speed conditions revealed that this motoric effect was not due to strategic effects. That is, there was no evidence for trial-by-trial guessing in which participants chose one of the two ambiguous alternatives. Rather, we found evidence for a multiple preparation hypothesis, that is, in the ambiguous precue conditions both response alternatives seem to be prepared in parallel. Confirming our earlier results, we also found multiple preparation in the direction precue condition, that is, a precued extension (flexion) movement led to preparing the movements in both response hands.

\section{REFERENCES}

Cardoso de Oliveira, S. (2002). The neuronal basis of bimanual coordination: Recent neurophysiological evidence and functional models. Acta Psychologica, 110, 139-159.

Coles, M. G. H. (1989). Modern mind-brain reading: Psychophysiology, physiology, and cognition. Psychophysiology, 26, 251-269.

Coles, M. G. H., Gratton, G., \& Donchin, E. (1988). Detecting early communication: Using measures of movement-related potentials to illuminate human information processing. Biological Psychology, 26, 69-89.
De Jong, R., Wierda, M., Mulder, G., \& Mulder, L. J. M. (1988). Use of partial stimulus information in response processing. Journal of Experimental Psychology: Human Perception and Performance, 14, 682-692.

Eimer, M. (1998). The lateralized readiness potential as an on-line measure of selective response activation. Behavior Research Methods, Instruments, \& Computers, 30, 146-156.

Goodman, D., \& Kelso, J. A. S. (1980). Are movements prepared in parts? Not under compatible (naturalized) conditions. Journal of Experimental Psychology: General, 109, 475-495. 
Hillyard, S. A., \& Münte, T. F. (1984). Selective attention to color and location: An analysis with event-related brain potentials. Perception \& Psychophysics, 36, 185-198.

Jentzsch, I., \& Leuthold, H. (2002). Advance movement preparation of eye, foot, and hand: A comparative study using movement-related brain potentials. Cognitive Brain Research, 14, 201-217.

Keele, S. W. (1981). Behavioral analysis of movement. In V. B. Brooks (Ed.), Handbook of physiology, section 1, volume 2. Motor control (pp. 1391-1414). Bethesda, MD: American Physiological Society.

Larish, D. D. (1986). Influence of stimulus-response translations on response programming: Examining the relationship of arm, direction, and extent of movement. Acta Psychologica, 61, 53-70.

Larish, D. D., \& Frekany, G. A. (1985). Planning and preparing expected and unexpected movements: Reexamining the relationship of arm, direction, and extend movements. Journal of Motor Behavior, 17, 168-189.

Lépine, D., Glencross, D., \& Requin, J. (1989). Some experimental evidence for and against a parametric conception of movement programming. Journal of Experimental Psychology: Human Perception and Performance, 15, 347-362.

Leuthold, H., \& Jentzsch, I. (2001). Neural correlates of advance movement preparation: A dipole source analysis approach. Cognitive Brain Research, 12, 207-224.

Leuthold, H., \& Jentzsch, I. (2002a). Distinguishing neural sources of movement preparation and execution: An electrophysiological analysis. Biological Psychology, 60, 173-198.

Leuthold, H., \& Jentzsch, I. (2002b). Spatio-temporal source localisation reveals involvement of medial premotor areas in movement reprogramming. Experimental Brain Research, 144, 178-188.

Leuthold, H., Sommer, W., \& Ulrich, R. (1996). Partial advance information and response preparation: Inferences from the lateralized readiness potential. Journal of Experimental Psychology: General, 125, 307-323.

MacKay, W. A., \& Bonnet, M. (1990). CNV, stretch reflex and reaction time correlates of preparation for movement direction and force. Electroencephalography and Clinical Neurophysiology, 76, 47-62.

Miller, J., \& Hackley, S. A. (1992). Electrophysiological evidence for temporal overlap among contingent mental processes. Journal of Experimental Psychology: General, 121, 195-209.

Miller, J., Patterson, T., \& Ulrich, R. (1998). Jackknife-based method for measuring LRP onset latency differences. Psychophysiology, 35, 99115.

Mordkoff, J. T., \& Gianaros, P. J. (2000). Detecting the onset of the lateralized readiness potential: A comparison of available methods and procedures. Psychophysiology, 37, 347-360.

Müller-Gethmann, H., Rinkenauer, G., Stahl, J., \& Ulrich, R. (2000). Preparation of response force and movement direction: Onset effects on the lateralized readiness potential. Psychophysiology, 37, 507-514.

Nicoletti, R., Umiltà, C., Tressoldi, E. P., \& Marzi, C. A. (1988). Why are left-right spatial codes easier to form than above-below ones? Perception \& Psychophysics, 43, 287-292.

Oldfield, R. C. (1971). The assessment and analysis of handedness: The Edinburgh Inventory. Neuropsychologia, 9, 97-113.

Oostenveld, R., Praamstra, P., Stegeman, D. F., \& van Oosterom, A. (2001). Overlap of attention and movement-related activity in lateralized event-related brain potentials. Clinical Neurophysiology, $112,477-484$.

Osman, A., \& Moore, C. M. (1993). The locus of dual-task interference: Psychological refractory effects on movement-related brain poten- tials. Journal of Experimental Psychology: Human Perception and Performance, 19, 1292-1312.

Osman, A., Moore, C., \& Ulrich, R. (1995). Bisecting RT with lateralized readiness potentials: Precue effects after LRP onset. Acta Psychologica, 90, 111-127.

Praamstra, P., Stegeman, D. F., Horstink, M. W. I. M., \& Cools, A. R. (1996). Dipole source analysis suggests selective modulation of the supplementary motor area contribution to the readiness potential. Electroencephalography and Clinical Neurophysiology, 98, 468-477.

Rohrbaugh, J., \& Gaillard, A. W. K. (1983). Sensory and motor aspects of the contingent negative variation. In A. W. K. Gaillard \& W. Ritter (Eds.), Tutorials in event-related potential research: Endogenous components (pp. 269-310). Amsterdam: Elsevier.

Rosenbaum, D. A. (1980). Human movement initiation: Specification of arm, direction and extent. Journal of Experimental Psychology: General, 109, 444-474

Rosenbaum, D. A. (1983). The movement precuing technique: Assumptions, applications, and extensions. In R. A. Magill (Ed.), Memory and control of action (pp. 231-274). Amsterdam: North-Holland.

Rosenbaum, D. A. (1991). Human motor control. San Diego, CA: Academic Press.

Rosenbaum, D. A., \& Kornblum, S. (1982). A priming method for investigating the selection of motor responses. Acta Psychologica, 51, $223-243$.

Sanders, A. (1980). Stage analysis of reaction processes. In G. E. Stelmach \& J. Requin (Eds.), Tutorials of motor behavior (pp. 331354). New York: North-Holland.

Schwarzenau, P., Falkenstein, M., Hoormann, J., \& Hohnsbein, J. (1998). A new method for the estimation of the onset of the lateralized readiness potential (LRP). Behavior Research Methods, Instruments, \& Computers, 30, 110-117.

Teichner, W. H., \& Krebs, M. J. (1974). Laws of visual choice reaction time. Psychological Review, 81, 75-98.

Ulrich, R., Leuthold, H., \& Sommer, W. (1998). Motor programming of response force and movement direction. Psychophysiology, 35, 721728

Ulrich, R., \& Miller, J. (2001). Using the jackknife-based scoring method for measuring LRP onset effects in factorial designs. Psychophysiology, 38, 816-827.

Van der Molen, M. W., Bashore, T. R., Halliday, R., \& Callaway, E. (1991). Chronopsychophysiology: Mental chronometry augmented by psychophysiological time markers. In J. R. Jennings \& M. G. H. Coles (Eds.), Handbook of cognitive psychophysiology: Central and autonomic nervous system approaches (pp. 9-178). New York: Wiley.

Vidal, F., Macar, F., \& Bonnet, M. (1996). The "short-long" reaction time effect in duration programming. Journal of Motor Behavior, 28, 359-370.

Wild-Wall, N., Sangals, J., Sommer, W., \& Leuthold, H. (2003). Are fingers special? Evidence about movement programming and preparation from event-related brain potentials. Psychophysiology, 40, 7-16.

Wright, D. L., Black, C., Park, J.-H., \& Shea, C. H. (2001). Planning and executing simple movements: Contributions of relative and absolute time specification. Journal of Motor Behavior, 33, 273-285.

(Received March 4, 2003; ACCEPTED July 22, 2003)

\section{APPENDIX}

Lateral presentation of stimuli is known to produce a sensoryinduced lateral ERP component with larger negativity contralateral to stimulus presentation side (e.g., Hillyard \& Münte, 1984). This asymmetric sensory activity has been reported to be maximally negative at parietal sites but also contributes activity measured at central electrodes due to volume conduction. To control for this sensory ERP activity and also to calculate its exact contribution to the LRP activity at central electrode sites, we decided to derive a dipole model of the early sensory activity and the later motor activity. Such a procedure gives us the opportunity to measure LRP onset latencies of motor-related activity by excluding early sensory activity.

Source models were determined by using Brain Electromagnetic Source Analysis (BESA2000). Symmetry constraints with respect to location and orientation were applied to lateral dipole pairs to limit the number of parameters to be estimated. Source models for lateralized motor-related activity were derived as described in more detail in studies by Praamstra, Stegeman, 

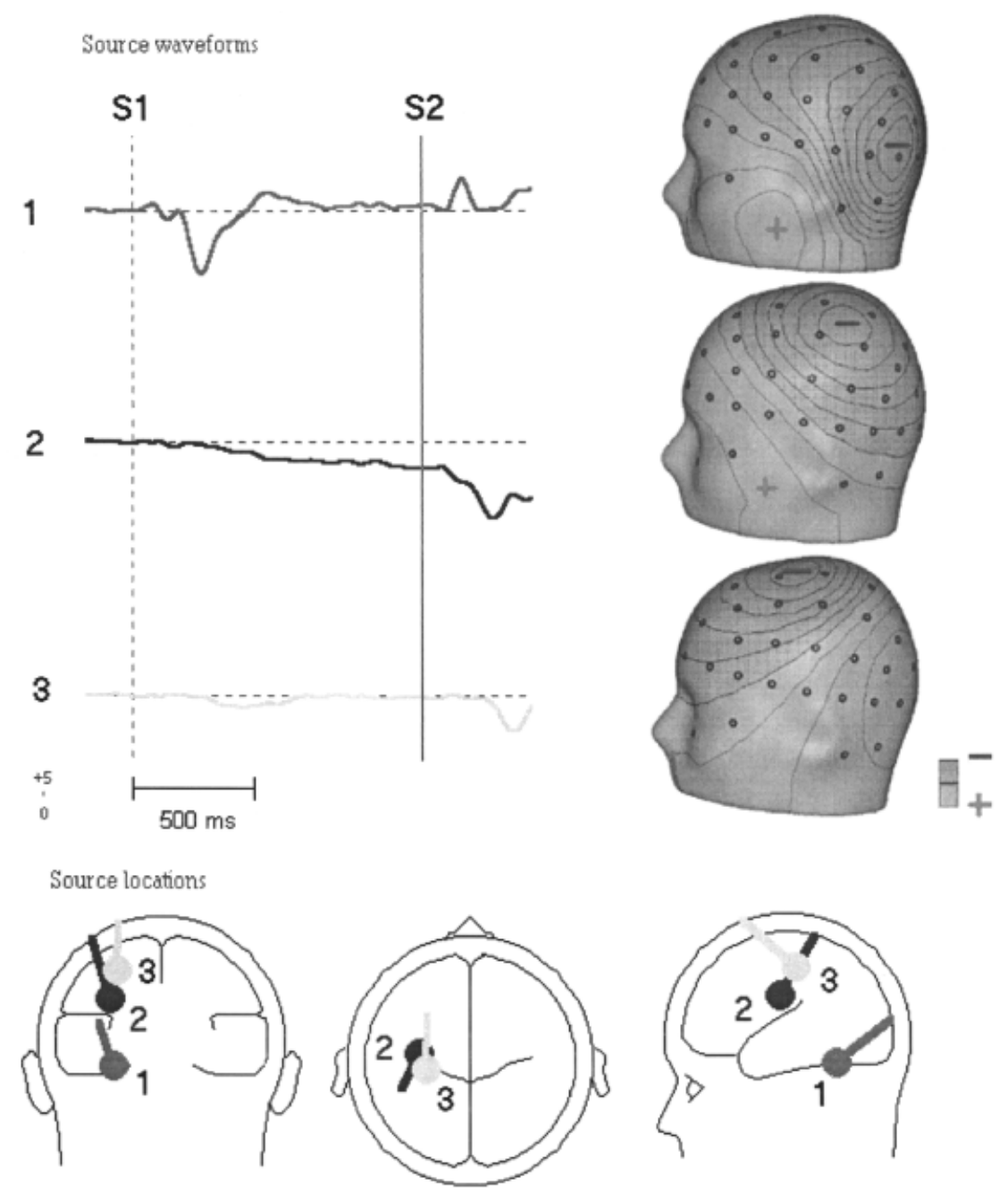

Figure 9. Dipole source model derived for the hand precue condition with source waveforms (top left), source distributions (top right), and dipole source locations (bottom).

Horstink, and Cools (1996) and Leuthold and Jentzsch (2001). L-ERPs were projected to one hemisphere and copied to the other hemisphere with polarity inverted. This procedure resulted in an antisymmetric scalp distribution of L-ERP activity (cf. Praamstra et al., 1996).

We fitted a source model for the parietal sensory activity using grand-average (across subjects) stimulus-locked waveforms of the hand precue condition. One dipole pair was fitted in an interval $250-300 \mathrm{~ms}$ relative to precue onset, where the parietal activity showed maximal amplitudes. As shown in Figure 9 (Dipole 1), the sources were located in occipito-temporal brain regions, nicely conforming with simulation results by Oostenveld et al. (2001). To derive a dipole model for the movement-related lateralization, response-synchronized grand averages for the hand precue were used and the dipoles representing MI (Dipole 2) and reafferential activity (Dipole 3) were fitted using a sequential fitting strategy (see Leuthold \& Jentzsch, 2001). While fitting these two dipole pairs, the dipole pair derived earlier in the S-locked data was included in the model but only allowed to vary in dipole source strength with both the location and the orientation parameters held fixed. The resulting three-pair dipole model is shown in Figure 9 (only the dipoles in one hemisphere are shown).

To estimate the onset of the "corrected" LRP motor activity, we projected the dipole model into the jackknife-averaged data (see Method section) and measured the onset of the source activity in the MI dipole (Dipole 2 in Figure 9) using the same criteria as for the LRP onset measurement.

Results. ANOVA of the S-LRP interval values for the different onset criteria and baselines using the dipole-corrected waveforms are shown in Table 1 (right). The duration of the S-LRP interval (values given for the $50 \%$ criterion) does not differ for the noninformative precue $(248 \mathrm{~ms})$, direction precue $(253 \mathrm{~ms})$, and ambiguous precue $(252 \mathrm{~ms})$ with any of the four onset criteria. In contrast to the results for the S-LRP intervals, clear precuing effects were found in the LRP-R interval (see Table 2, right). Motoric processing time (values given for the $50 \%$ criterion) was longer when no advance information about the upcoming movement was provided (noninformative precue $=87 \mathrm{~ms}$ ), compared to the direction precue $(69 \mathrm{~ms})$ and the ambiguous precue $(73 \mathrm{~ms})$. 